\title{
Image Enhancement under Data-Dependent Multiplicative Gamma Noise
}

\author{
Jidesh Pacheeripadikkal $^{1}$ and Bini Anattu ${ }^{2}$ \\ ${ }^{1}$ Department of Mathematical and Computational Sciences, National Institute of Technology, Karnataka 575025, India \\ ${ }^{2}$ Department of Electronics and Communications Engineering, National Institute of Technology, Karnataka 575025, India
}

Correspondence should be addressed to Jidesh Pacheeripadikkal; jidesh@nitk.ac.in

Received 13 February 2014; Accepted 19 May 2014; Published 1 June 2014

Academic Editor: Christian W. Dawson

Copyright (C) 2014 J. Pacheeripadikkal and B. Anattu. This is an open access article distributed under the Creative Commons Attribution License, which permits unrestricted use, distribution, and reproduction in any medium, provided the original work is properly cited.

\begin{abstract}
An edge enhancement filter is proposed for denoising and enhancing images corrupted with data-dependent noise which is observed to follow a Gamma distribution. The filter is equipped with three terms designed to perform three different tasks. The first term is an anisotropic diffusion term which is derived from a locally adaptive $p$-laplacian functional. The second term is an enhancement term or a shock term which imparts a shock effect at the edge points making them sharp. The third term is a reactive term which is derived based on the maximum a posteriori (MAP) estimator and this term helps the diffusive term to perform a Gamma distributive data-dependent multiplicative noise removal from images. And moreover, this reactive term ensures that deviation of the restored image from the original one is minimum. This proposed filter is compared with the state-of-the-art restoration models proposed for data-dependent multiplicative noise.
\end{abstract}

\section{Introduction}

Image restoration is an important activity in image processing. Many different kinds of degradation models have been discussed in the recent literature. The degradation due to an additive data independent noise was explored extensively in the image denoising literature; see [1-3] for details. Further the degradations due to the combined effect of random noise and linear shift invariant blur are also studied elaborately; see [4-7] for further details. Another category of degradation that is discussed in the recent literatures is the model with multiplicative datadependent noise with linear shift-invariant blur. Nevertheless, a solution to data-dependent noise model should practically consider the noise distribution as well [8]. Different noise distributions considered under a multiplicative data-dependent noise set-up include Gamma, Poisson, and Gaussian distributions; refer to [8-10] for further details.

\section{PDE Models for Multiplicative Noise}

A common representation for a multiplicative denoising model, found in the image processing literature (assuming a shift-invariant nature of the linear blurring operator), is

$$
u_{0}=k * u \times n
$$

where $k$ is a linear shift invariant blurring kernel, “*” denotes a linear convolution operator, and $n$ is a multiplicative datadependent noise. The distribution of the noise intensity varies with reference to the application under consideration.

The first kind of model that was proposed for image restoration assuming a Gaussian multiplicative noise was RLO model by Lions et al. in [10]. The diffusion equation for the model is

$$
\begin{aligned}
u_{t}= & \operatorname{div} \frac{\nabla u}{|\nabla u+\beta|}-\lambda k *\left(\frac{u_{0}}{(k * u)^{2}}\right)\left(\frac{u_{0}}{(k * u)-1}\right) \\
& -\mu k *\left(\frac{u_{0}}{(k * u)^{2}}\right),
\end{aligned}
$$


where $\lambda$ and $\mu$ are the parameters which control the regularization and the data fitting characteristics of the filter. These parameters are updated dynamically during the evolution process. We use the notation $u_{t}$ to represent the partial derivative $\partial u / \partial t$ and div denotes a divergence operator. We invite readers to refer to [10] for details on the update of these parameters and the theoretical analysis of the model. The solution to this model is defined in the space of bounded variations. The model is well suited for data independent Gaussian multiplicative noise with mean 1 and variance $\sigma^{2}$. However, most of the imaging modalities that are in place today do not produce images with Gaussian multiplicative noise. Many of these models produce speckled images whose intensities are Gamma distributed ultrasound and satellite images are good examples.

Aubertt and Aujol [8] proposed a model (AA model) for restoring images corrupted by multiplicative Gamma noise. This model adopts the diffusion term from the total variation (TV) regularization model in [6] (ROF) and the reactive term is derived based on the Bayesian maximum a posteriori (MAP) estimator. The Euler Lagrange equation for this model is

$$
u_{t}=-\operatorname{div}\left(\frac{\nabla u}{|\nabla u|}\right)+\lambda k * \frac{\left(k * u-u_{0}\right)}{(k * u)^{2}} .
$$

Here $\lambda$ is a regularization parameter. And taking negative of the above equation gives the gradient descent solution:

$$
u_{t}=\operatorname{div}\left(\frac{\nabla u}{|\nabla u|}\right)-\lambda k * \frac{\left(k * u-u_{0}\right)}{(k * u)^{2}} .
$$

Since the diffusion term (the first term in the above equation) is adopted from the TV model, the diffusive behavior of this filter is quite similar to the ROF model; therefore, the process results in formation of staircases in restored images. The reactive term (second term in the above equation) is conditionally convex, and therefore uniqueness of the solution cannot be proven conclusively. Some modifications are suggested for this model in the recent literature. In [11] the authors propose a Weberized diffusive term for improving the intensity of the filtered image and thereby enhancing the visual quality of the output. We recall the Weber's low; the intensity increment in the restored data is directly proportional to mean intensity of the background (see [11] for details). However, this model retains the reactive term in AA model and therefore the solution may not be unique. Another remarkable modification to AA model is the one proposed in [12]. In this model, the data fitting term is modified so that the functional remains convex under all conditions. Therefore, a unique solution is guaranteed under a gradient descent set-up. Nevertheless, the total variation based diffusive term may eventually form piecewise constant regions in the filtered output. There are few improvements suggested for the AA model to overcome the difficulties due to the presence of second-order TV diffusion term; refer to $[13,14]$ for details. Moreover, none of the models discussed above could really enhance the image features while denoising images. However, in many applications enhancement of images is vital.

\section{Image Enhancement Models}

Though the models discussed above are capable of restoring images from their blurred and noisy observations, they lack the capability to enhance the images. Image enhancement is an essential step in many image processing applications. Shock filters were introduced in the literature to enhance images from their blurred observations. A classical shock filter introduced by Osher and Rudin in [15] follows the EulerLagrange equation:

$$
u_{t}=-\operatorname{sign}\left(u_{\eta \eta}\right)|\nabla u|,
$$

where sign denotes the mathematical sign function, which takes the values from the set $\{-1,0,1\}$. This filter enhances the high frequency components present in the images; however, the noise components also get enhanced, consequently. Therefore, a preprocessing on the input image becomes inevitable.

In another work, Alvarez and Mazorra [16] proposed a diffusion coupled shock filter. This model enhances and denoises simultaneously, so that the noise features do not blow up in the restored version of the image. The model is formulated as

$$
u_{t}=-\operatorname{sign}\left(u_{\eta \eta}\right)|\nabla u|+\lambda u_{\xi \xi},
$$

were $\xi$ denotes the direction normal to the gradient or parallel to the level line and $\lambda$ is a regularization parameter. The term $u_{\xi \xi}$ is mean curvature of the level curve. The first term signifies a shock filter and the second one is a diffusion term. This model is quite effective in enhancing images in case of noise intervention. There are quite a few modifications suggested for this model, as well. The modifications are suggested for both diffusion and the shock terms. We invite readers to refer to [17-19] for details.

All these facts discussed above motivated us to propose a filter which enhances the edge features while restoring the images from their blurred and noisy observations, especially when the noise is multiplicative and Gamma distributed. The detailed mathematical formulation and experimental results are given in succeeding sections.

\section{The Proposed Model}

The energy functional for the new model is

$$
\min _{u} E(u)=\int_{\Omega}|\nabla u|^{p(s)} d \Omega+\lambda \int_{\Omega}\left(\log u+\frac{u_{0}}{u}\right) d \Omega,
$$

where $u_{0}$ is the observed image, $\Omega$ denotes the image domain, and the function $p(s)$ (where $s$ denotes $|\nabla u|$ ) is defined as

$$
p(s)=1+\frac{1}{1+s^{2}} .
$$

This functional is a modified form of the functional proposed by Aubertt and Aujol in [8] and Blomgren et al. in [20]. The reactive term of the proposed model is borrowed from AA model, which is in fact derived based on the Bayesian 
MAP estimator. The details of the $p$-laplacian diffusion term in the above equation can be found in [20]. The proposed functional is not purely convex and the details are provided in the Appendix to follow. The Euler-Lagrange equation can be derived as

$$
u_{t}=-\nabla \cdot\left(\frac{\nabla u}{|\nabla u|^{2-p(s)}}\right)+\lambda_{2} \frac{\left(u-u_{0}\right)}{u^{2}}
$$

The solution to this equation is derived at the steady state (when $u_{t}=0$ ). Here we note that this evolution PDE does not have any enhancing capability on its own. Nevertheless, enhancement comes handy in many imaging problems because of the common device artifacts resulting in blurred versions of the captured images. Therefore, we modify the PDE defined in (9) to incorporate the enhancing capabilities as well. The new proposed PDE takes the following form:

$$
\begin{aligned}
u_{t}= & -\nabla \cdot\left(\frac{\nabla u}{|\nabla u|^{2-p(s)}}\right) \\
& -\lambda_{1} \frac{2}{\pi} \arctan \left(G_{\sigma} * u_{\eta \eta} k(t)\right)|\nabla u| \\
& +\lambda_{2} \frac{\left(u-u_{0}\right)}{u^{2}}
\end{aligned}
$$

where $\lambda_{1}$ and $\lambda_{2}$ are the parameters that are evaluated experimentally. Here $\lambda_{2}$ is evaluated as $\lambda_{2}=0.5 / \sigma^{2}$, where $\sigma$ is the noise variance and $\lambda_{1}$ is assigned a value 0.02 experimentally to get the desired enhancement. The term $G_{\sigma} u$ stands for a Gaussian convolved version of the image function $u$ and $\sigma$ here denotes the standard deviation of the Gaussian function used for smoothing the image. The diffusion term causes a denoising effect, whereas the shock term (the second term in the above equation) is responsible for the enhancement property of the filter (see [18] for details) and the reactive term ensures the deviation from the actual solution to be minimal (so that restoration is more accurate). The parameters $\lambda_{1}$ and $\lambda_{2}$ control the enhancement and the denoising behavior of the filter. The function $p(\cdot)$ as already defined in (8) depends on the gradient of the image function. In the regions where gradient value is zero or in the constant intensity regions the function takes a value 2 and in high gradient regions the functions assume a value 1 and in all other regions it assumes a value between 1 and 2 . Since the diffusion process is driven by the value of the function $p(\cdot)$, this diffusion term is commonly known as adaptive $p$ laplacian term. The $p$-laplace term reduces the staircase effect due to the prompt switching of the filter between norms in the range [1-2]. In order to avoid the shock components being active during the initial phases of evolution, a timedependent function $k(t)$ is used in the shock term. This function helps diffusion process to dominate the evolution during the initial stages of the evolution process; refer to $[18,19]$ for details of this function. Here we assume that the noise variance and the blurring function are known in advance and the noise distribution is assumed to be Gamma.

\section{Numerical Implementation}

Explicit central difference schemes are employed for representing the finite differences of the terms in the proposed filter in (10) except the shock term, for which upwind [21] scheme is applied. Discretization of diffusion equation is

$$
\begin{gathered}
u_{x x}\left(\delta+u_{y}^{2}\right)-2 u_{x} \times u_{y} \times u_{x y} \\
+\frac{u_{y y}\left(\delta+u_{x}^{2}\right)}{\left(\delta+u_{x}^{2}+u_{y}^{2}\right)^{4-(p(s)) / 2}},
\end{gathered}
$$

where $\delta$ is a small positive value used to avoid "division by zero" in homogeneous intensity regions. The term $|\nabla u|$ in the shock term is discretized using the upwind scheme proposed in [21] to make the process stable

$$
\|\nabla u\|=\sqrt{D_{x}^{2}+D_{y}^{2}}
$$

where $D_{x}=\operatorname{minmod}\left(u_{x}^{+}(x, y), u_{x}^{-}(x, y)\right), D_{y}=\operatorname{minmod}\left(u_{y}^{+}\right.$ $\left.(x, y), u_{y}^{-}(x, y)\right)$, and the minmod operator is defined as

$$
\operatorname{minmod}(x, y)= \begin{cases}\min (\|x\|,\|y\|) & \text { if } x y>0 \\ 0 & \text { Otherwise }\end{cases}
$$

The first-order forward, backward, and central difference operators are as follows:

$$
\begin{aligned}
& u_{x}^{+}(x, y)=u(x+1, y)-u(x, y), \\
& u_{x}^{-}(x, y)=u(x, y)-u(x-1, y), \\
& u_{x}(x, y)=\frac{\left(u_{x}^{+}+u_{x}^{-}\right)}{2}, \\
& u_{y}^{+}(x, y)=u(x, y+1)-u(x, y), \\
& u_{y}^{-}(x, y)=u(x, y)-u(x, y-1), \\
& u_{y}(x, y)=\frac{\left(u_{y}^{+}+u_{y}^{-}\right)}{2} .
\end{aligned}
$$

Here the super scripts +/- denote the forward and backward finite differences, respectively, and nonsuperscripted symbols stand for the finite central differences. Finally the diffusion equation is discretized using the above-mentioned finite difference schemes.

\section{Experimental Results}

We have tested various images with different characteristics; however, the visual results are provided only for two test images "peppers" and "Lena" to maintain the brevity in explanation. However, we confirm that the model works for other images as well. These test images are chosen in such a way that they belong to two different classes. The image "pepper" is a low textured cartoon type image with homogeneous intensity regions and image "Lena" is a partially textured one. The 
(1) Input the noisy image $u_{0}$ corrupted by out-of-focus blur and data-dependent multiplicative Gamma noise.

(2) Calculate the regularization weight $\lambda_{2}$ as $0.5 / \sigma_{n}{ }^{2}$ where $\sigma_{n}$ is the noise standard deviation and $\lambda_{1}=0.02$ (this was set empirically).

(3) Specify the stopping threshold value $t$.

(4) Select a time step of $\Delta t=0.1$ for the PDE evolution.

(5) Initialize the current restored image $u_{r}^{n}=0$ and $u_{n+1}^{r}=u_{0}$

(6) while $\left(\left\|u_{r}^{n+1}-u_{r}^{n}\right\| /\left\|u_{r}^{n}\right\|\right)>t$ do,

(7) Assign $u_{r}^{n}=u_{r}^{n+1}$

(8) Evaluate $u_{r}^{n+1}$ using the PDE defined in (10).

(9) end while

Algorithm 1: Image enhancement filter.

images are normalized to the range [0-1] in our experiments. The blurring artifact is simulated using a spatially invariant Gaussian kernel with $\sigma$ value 3. We transform the image to the Fourier domain and multiply it with the frequency response of the Gaussian kernel and transform the image back to the spatial domain to get the blurred version of the input image. Further, the multiplicative Gamma noise (with unit mean) is added with different noise variances to the images under testing. We have generated the Gamma noise (with the specified variance) using a function written in MATLAB. We also note that all the methods considered in this work are simulated (programmed) using MATLAB 7.9 (R2009b) on Linux platform. The process is iterated till the error in consecutive iterations becomes less than a threshold value. The overall algorithm for the model is summarized in Algorithm 1.

The visual results in Figures 1 and 2 for the test images "peppers" and "Lena" show the performances of various restoration methods under a multiplicative Gamma noise set-up. Here we consider the multiplicative noise restoration models AA [8] and RLO [10], for demonstrating and comparing the restoration capacities of various filters under consideration and the one proposed in this paper. As evident from these figures, the RLO model is not that effective for the multiplicative Gamma noise, as it is designed mainly for handling Gaussian distributed multiplicative noise. AA model performs better than RLO model; nevertheless, it forms staircases on the sharp edges when it eventually reaches to the steady state, as evident from Figures 1(c), 1(g), 2(c) and $2(\mathrm{~g})$. The proposed model is capable of enhancing and restoring images from their noisy observations without causing any staircase effect; see Figures $1(\mathrm{~d}), 1(\mathrm{~h}), 2(\mathrm{~d})$, and 2(h), for a comparative analysis. Moreover, the deblurring/ deconvolution capability of the proposed model is evident in these figures. We have tested the models for various noise variance values (the results for two different noise variances and two different blurs are provided in Figures 1 and 2) and different quantity of blurs. In all these different degradation scenarios, the proposed model is observed to perform at a better scale in terms of visual representation compared to the other contemporary and relevant methods discussed in the literature.

We perform a quantitative study of various restoration models including the one proposed in this work, using
TABLE 1: Statistical measures $\{$ PFOM, SSIM $\}$ plotted for different images for different models.

\begin{tabular}{lccc}
\hline Images & RLO & AA & The proposed model \\
\hline Lena & $\{0.73,0.71\}$ & $\{0.79,0.76\}$ & $\{0.88,0.86\}$ \\
Cameraman & $\{0.71,0.68\}$ & $\{0.79,0.74\}$ & $\{0.91,0.88\}$ \\
Peppers & $\{0.74,0.71\}$ & $\{0.81,0.77\}$ & $\{0.93,0.91\}$ \\
Woman & $\{0.73,0.69\}$ & $\{0.80,0.79\}$ & $\{0.90,0.87\}$ \\
Baboon & $\{0.68,0.67\}$ & $\{0.73,0.71\}$ & $\{0.82,0.78\}$ \\
\hline
\end{tabular}

standard statistical measures like Pratt's figure of merit (PFOM) [22] and structural similarity index (SSIM) [23]. Table 1 is dedicated for comparing the methods under consideration in terms of these two statistical measures. From this table we may infer that the proposed model has a better performance rate in terms of these statistical measures compared to other methods under consideration. The PFOM value signifies the edge preservation capability of the filter under consideration. The values are in the range [0-1]; the value 1 stands for a perfect edge preservation. Finally, SSIM denotes the structure similarity between the restored and the original images. This value also lies in the range [0-1]. And the value approaches 1 as the structure preservation approaches the perfection level. The numerical values tabulated for the two different statistical measures prove beyond doubt that the proposed model outperforms other models considered here, in terms of edge and structure preservation. The figures shown are highly in favor of the noise removal and edge and structure preservation capability of the proposed model.

\section{Conclusion}

In this work a filter is proposed for enhancing the images while restoring them from their blurred and noisy observations. The images are assumed to be corrupted with multiplicative Gamma noise and shift invariant Gaussian blur. Further the presence of a switching $p$-laplacian diffusion term helps in reducing the staircase effect, which otherwise appears in all second-order nonlinear PDE models. The Experiments are performed both in terms of visual and quantitative statistical measures and all the results are in favor 


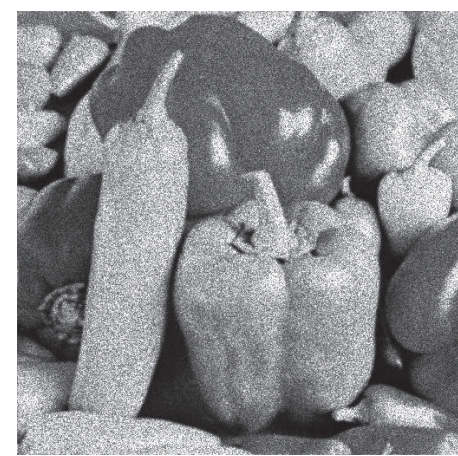

(a)

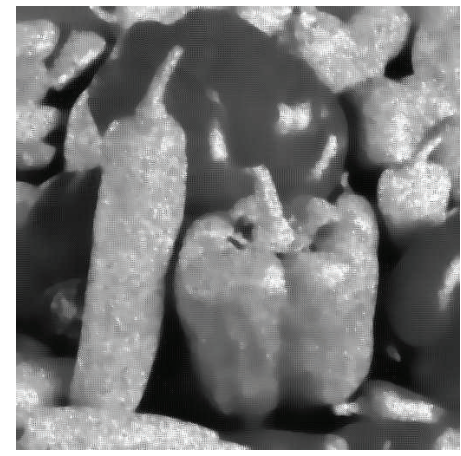

(c)

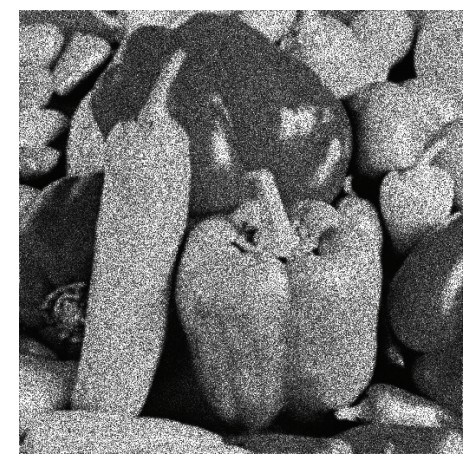

(e)

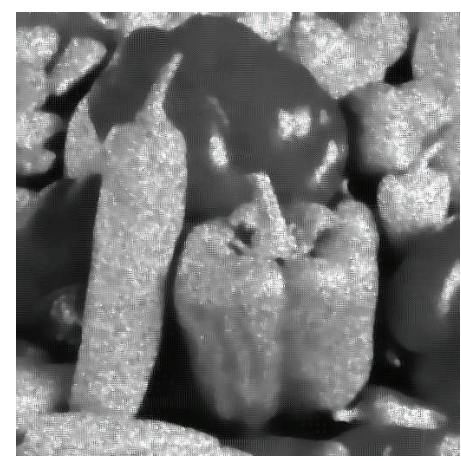

(g)

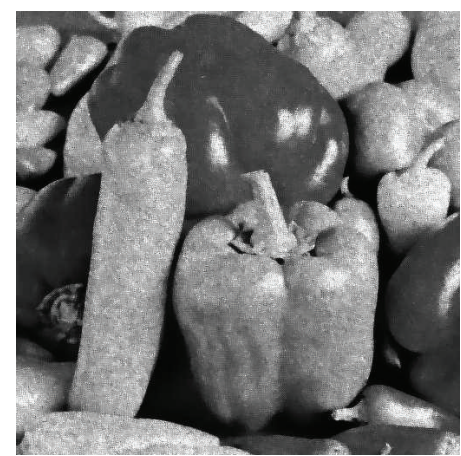

(b)

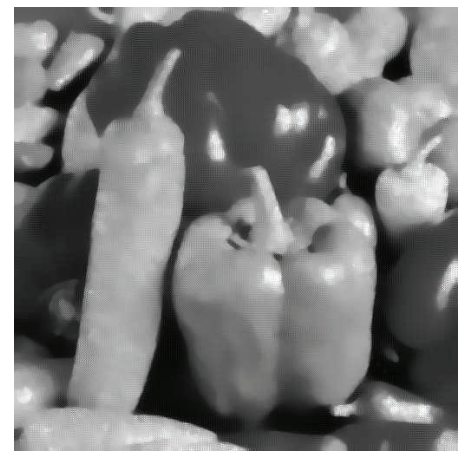

(d)

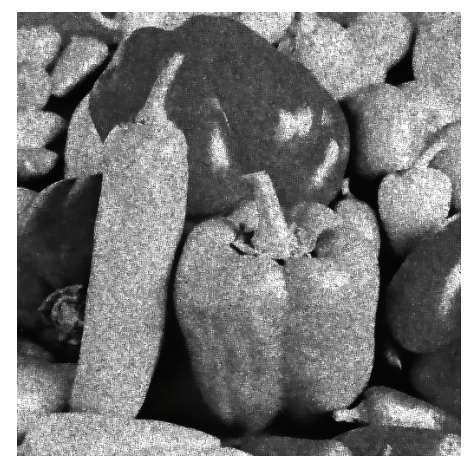

(f)

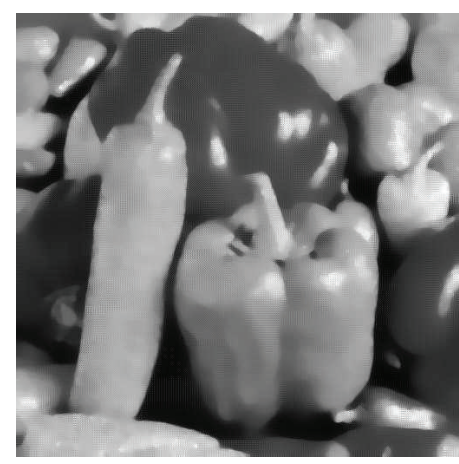

(h)

FIGURE 1: Images restored using various methods, images of "peppers": (a) and (e): noisy images with mean 1, noise variances 0.1 and 0.2 , and Gaussian blurs with kernel spread of 3 \& 4, respectively; (b) and (f) restored using RLO method [10]; (c) and (g) restored using AA filter [8]; (d) and (h) restored using the proposed method. 


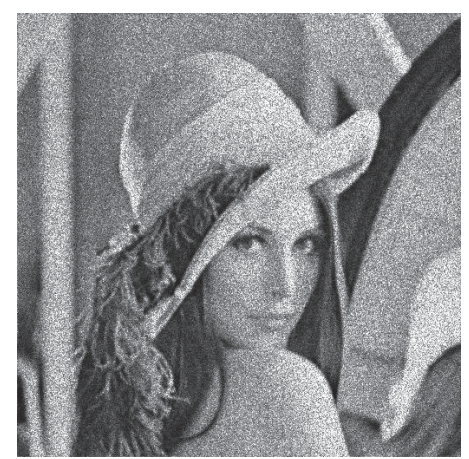

(a)

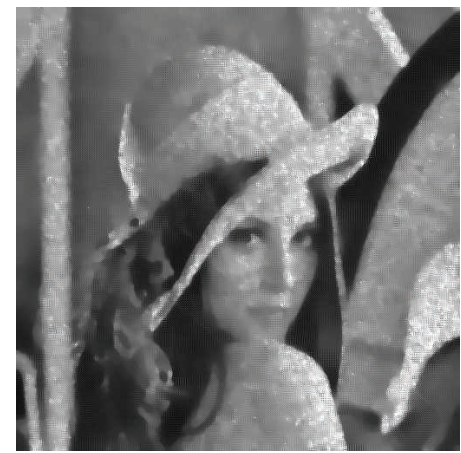

(c)

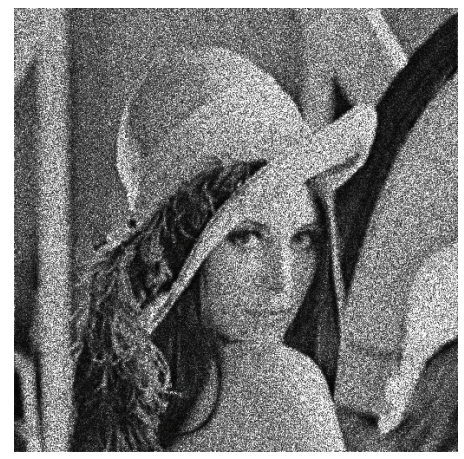

(e)

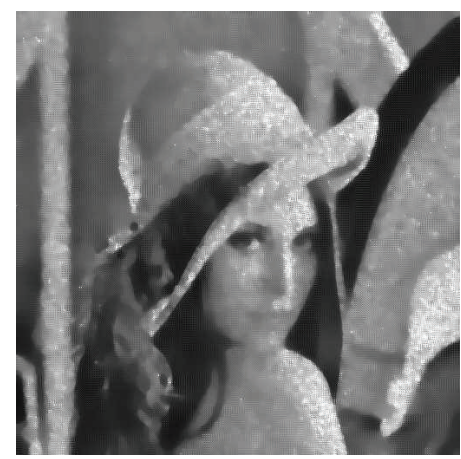

(g)

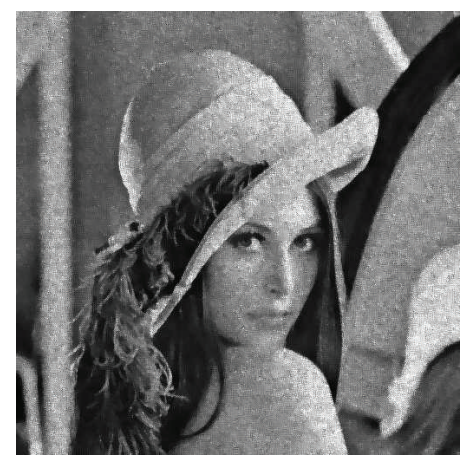

(b)

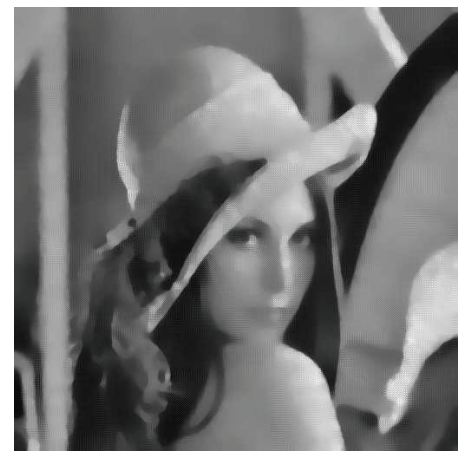

(d)

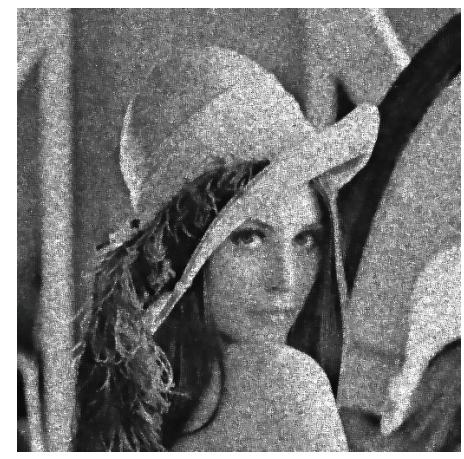

(f)

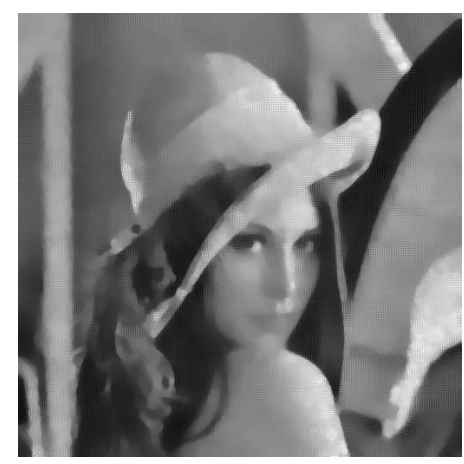

(h)

FIGURE 2: Images restored using various methods, images of "Lena": (a) and (e): noisy images with mean 1, noise variances 0.1 and 0.2 , and Gaussian blurs with kernel spread of 3 \& 4, respectively; (b) and (f) restored using RLO method [10]; (c) and (g) restored using AA filter [8]; (d) and (h) restored using the proposed method. 
of the claim that the proposed model outperforms the other models considered in this work.

\section{Appendix}

\section{Convexity of the Functional}

The energy functional associated with the diffusion term of the proposed PDE is

$$
\phi(u)=\int_{\Omega}|\nabla u|_{T V}^{p(s)} d x d y
$$

The EL equation associated with the PDE is derived as

$$
u_{t}=-\nabla \cdot \frac{\left(\phi^{\prime}(u) \nabla u\right)}{|\nabla u|}
$$

Therefore,

$$
u_{t}=-\nabla \cdot \frac{\left(|\nabla u|^{p-2} \nabla u\right)}{|\nabla u|}
$$

or

$$
u_{t}=-\nabla \cdot \frac{\nabla u}{\left(|\nabla u|^{2-p(s)}\right)}
$$

We can observe that the second derivative of the functional $\phi(x)=x^{p}$ is $\phi^{\prime \prime}(x)=p \times(p-1) \times \phi^{p-2}$. Therefore, $p \geq 1$; the functional is convex and strictly convex for $p>1$. The reactive term of the functional is $\log u+u_{0} / u$ and the second derivative of the term is $\left(1 / u^{3}\right)\left(2 u_{0}-u\right)$. For this term to be convex (in the strict sense), the necessary condition is $0<u<$ $2 u_{0}$. Therefore, the functional is conditionally convex.

\section{Conflict of Interests}

The authors declare that there is no conflict of interests regarding the publication of this paper.

\section{Acknowledgment}

The authors would like to thank all the colleagues at National Institute of Technology, Karnataka for the help and support extended by them.

\section{References}

[1] P. Perona and J. Malik, "Scale-space and edge detection using anisotropic diffusion," IEEE Transactions on Pattern Analysis and Machine Intelligence, vol. 12, no. 7, pp. 629-639, 1990.

[2] H. Kim, V. R. Calvert, and S. Kim, "Preservation of fine structures in pde-based image denoising," Advances in Numerical Analysis, vol. 2012, Article ID 750146, 19 pages, 2012.

[3] X. Li, L. Shao, R. Yan, and Y. Liu, "From heuristic optimization to dictionary learning: a review and comprehensive comparison of image denoising algorithms," IEEE Transactions on Cybernetics, 2013.
[4] D. Bertaccini, R. H. Chan, S. Morigi, and F. Sgallari, "An adaptive norm algorithm for image restoration," Lecture Notes in Computer Science, vol. 6667, pp. 194-205, 2012.

[5] R. H. Chan, A. Lanza, S. Morigi, and F. Sgallari, "An adaptive strategy for the restoration of textured images using fractional order regularization," Numerical Mathematics, vol. 6, no. 1, pp. 276-296, 2013.

[6] L. I. Rudin, S. Osher, and E. Fatemi, "Nonlinear total variation based noise removal algorithms," Physica D: Nonlinear Phenomena, vol. 60, no. 1-4, pp. 259-268, 1992.

[7] A. Marquina and S. Osher, "Explicit algorithms for a new time dependent model based on level set motion for nonlinear deblurring and noise removal," SIAM Journal on Scientific Computing, vol. 22, no. 2, pp. 387-405, 2001.

[8] G. Aubertt and J.-F. Aujol, "A variational approach to removing multiplicative noise," SIAM Journal on Applied Mathematics, vol. 68, no. 4, pp. 925-946, 2008.

[9] S. Setzer, G. Steidl, and T. Teuber, "Deblurring Poissonian images by split Bregman techniques," Journal of Visual Communication and Image Representation, vol. 21, no. 3, pp. 193-199, 2010.

[10] P. L. Lions, L. I. Rudin, and S. Osher, "Multiplicative denoising and deblurring: theory andalgorithms," in Geometric Level Set Methods in Imaging, Vision, and Graphics, pp. 103-120, Springer, 2003.

[11] L. Xiao, L.-L. Huang, and Z.-H. Wei, "Multiplicative noise removal via a novel variational model," Eurasip Journal on Image and Video Processing, vol. 2010, Article ID 250768, 2010.

[12] M. K. Ng, M. Huang, and Y. Y. W. Wen, "A new total variation method for multiplicative noise removal," SIAM Journal on Imaging Sciences, vol. 2, no. 1, pp. 22-40, 2009.

[13] P. Jidesh and A. A. Bini, "A complex diffusion driven approach for removing data-dependent multiplicative noise," in Proceedings of the Pattern Recognition and Machine Intelligence, vol. 8251 of Lecture Notes in Computer Science, pp. 284-289, 2013.

[14] P. Jidesh, "A convex regularization model for image restoration," Computers and Electrical Engineering, 2014.

[15] S. Osher and L. I. Rudin, "Feature-oriented image enhancement using shock filters," SIAM Journal on Numerical Analysis, vol. 27, no. 4, pp. 919-940, 1990.

[16] L. Alvarez and L. Mazorra, "Signal and image restoration using shock filters and anisotropic diffusion," SIAM Journal on Numerical Analysis, vol. 31, no. 2, pp. 590-605, 1994.

[17] G. Gilboa, N. A. Sochen, and Y. Y. Zeevi, "Regularized shock filters and complex diffusion," in Proceedings of the Computer Vision (ECCV'02), pp. 399-413, 2002.

[18] P. Jidesh and S. George, "Curvature driven diffusion coupled with shock for image enhancement/reconstruction," International Journal of Signal and Imaging Systems Engineering, vol. 4, no. 4, pp. 238-247, 2011.

[19] P. Jidesh and S. George, "Shock coupled fourth-order diffusion for image enhancement," Computers and Electrical Engineering, vol. 38, no. 5, pp. 1262-1277, 2012.

[20] P. Blomgren, T. F. Chan, and P. Mulet, "Extensions to total variation denoising," in Advanced Signal Processing: Algorithms, Architectures and Implementations VII, Proceedings of the SPIE, pp. 367-375, July 1997.

[21] S. Osher and J. A. Sethian, "Fronts propagating with curvaturedependent speed: algorithms based on Hamilton-Jacobi formulations," Journal of Computational Physics, vol. 79, no. 1, pp. 1249, 1988. 
[22] W. K. Pratt, Digital Image Processing, Wiley, 4th edition, 2007.

[23] Z. Wang, A. C. Bovik, H. R. Sheikh, and E. P. Simoncelli, "Image quality assessment: from error visibility to structural similarity," IEEE Transactions on Image Processing, vol. 13, no. 4, pp. 600612, 2004. 

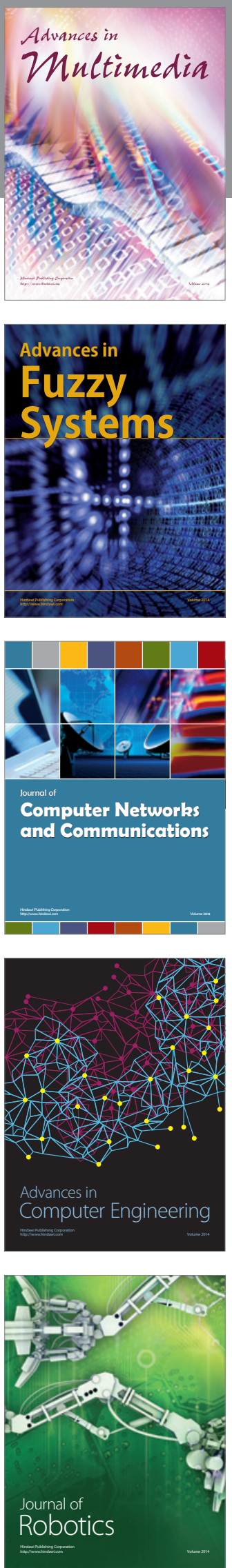

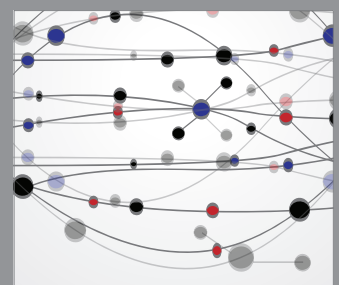

The Scientific World Journal
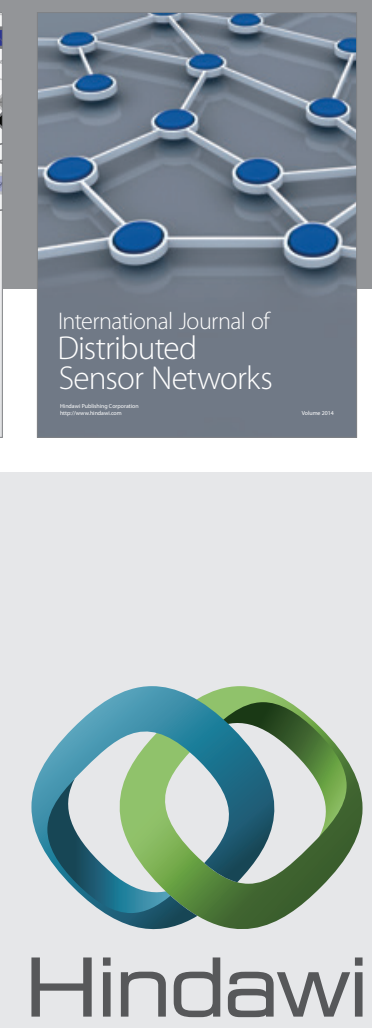

Submit your manuscripts at

http://www.hindawi.com
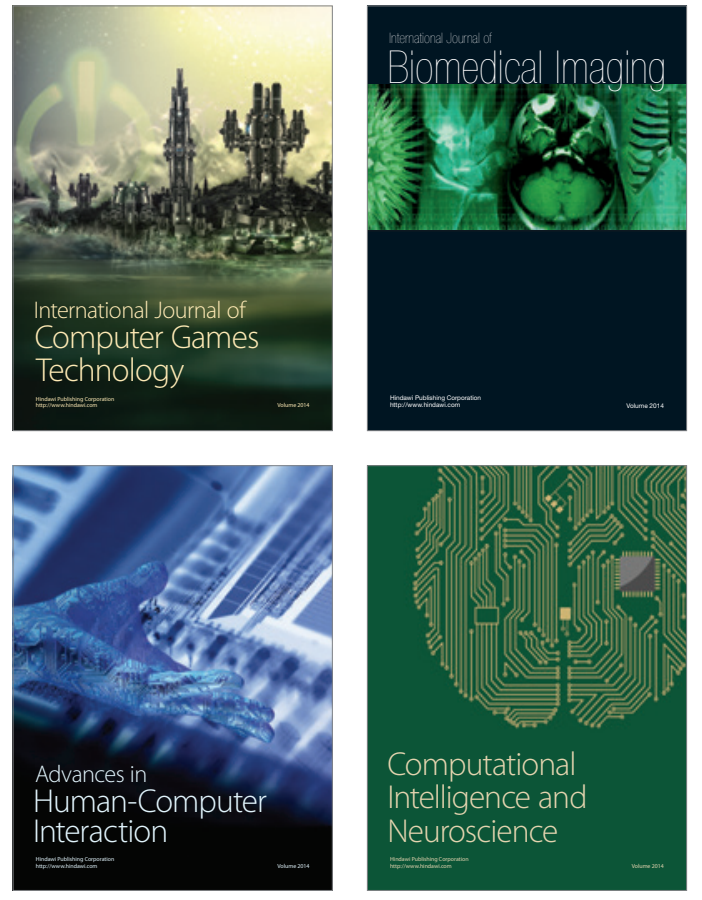
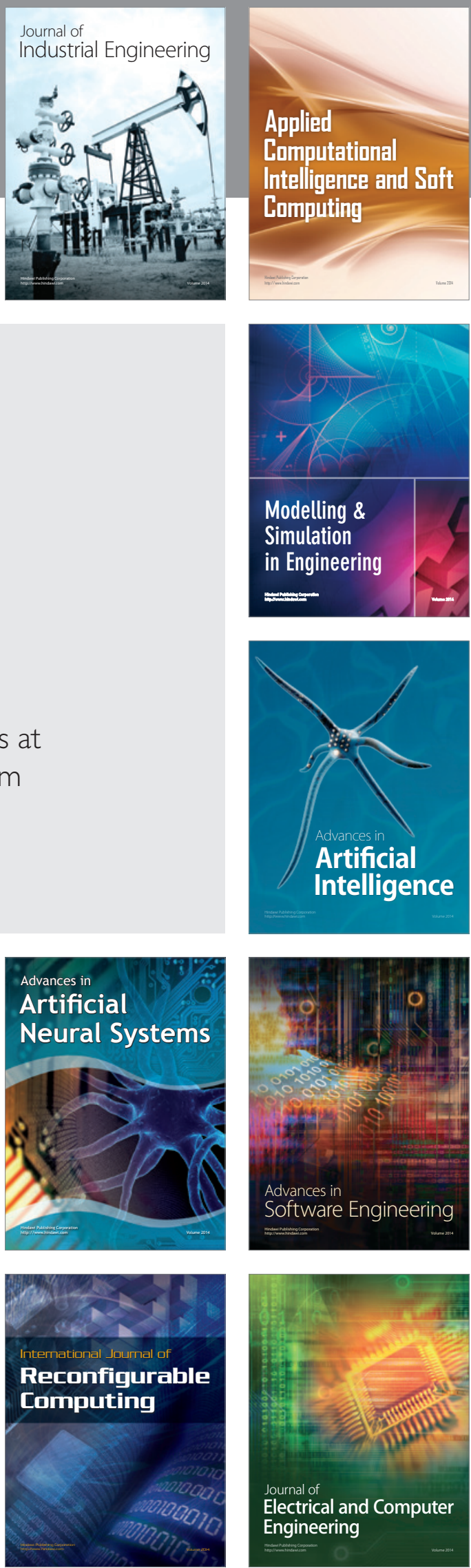